\title{
OUTCOME ANALYSIS OF EARLY ENDOMETRIAL CANCERS MANAGED BY ADJUVANT VAULT BRACHYTHERAPY- A SINGLE INSTITUTIONAL STUDY
}

\author{
Saravanan $S^{1}, P$. Vasanthamani ${ }^{2}$ \\ 1 Professor, Department of Radiotherapy, Government Royapettah Hospital and Kilpauk Medical College, Chennai, Tamilnadu, India. \\ ${ }^{2}$ Professor, Department of Obstetrics and Gynaecology, Government Royapettah Hospital and Kilpauk Medical College, Chennai,
} Tamilnadu, India.

\section{ABSTRACT}

\section{BACKGROUND}

Management of endometrial cancer in earlier stages have led to improved Overall Survival (OS) and Progression Free Survival (PFS). Therefore, vaginal brachytherapy (VBT) alone or along with EBRT can be used as adjuvant treatment in intermediate-risk disease (Stage IB G1-2 disease, stage IA G3 disease and stage IC G1-2 disease), with good results in terms of local control and toxicity.

\section{METHODS}

This single institute prospective analytical study included data of 306 patients who presented to Govt. Royapettah Hospital, attached to Govt. Kilpauk Medical College, after surgery, during the period 2012 - 2018. In this study, patients with endometrial cancer who underwent surgical management and adjuvant vaginal BT were included. This study has taken into consideration the following characteristics- age, histopathology, dose for each fraction, grading scale of toxicity used for each study, and follow-up time in both Treatment and Observed groups.

\section{RESULTS}

Median follow up was 49 months (Range 15 - 68) in the Treated Group Vs 40 months (range 12-36) in Observed Group. Number of patients who were disease free in the treated group $216(91 \%)$ and in observed group $54(85 \%)$. Patients with vault recurrence were only $12(0.5 \%)$ in Treated Group and $2(2.9 \%)$ in Observed Group. Patients with pelvic nodes recurrence were only $10(0.4 \%)$ in Treated Group and 2 (2.9\%) in Observed Group. Patients with distant metastases were only 12 (0.5\%) in the Treated Group and $2(2.9 \%)$ in the Observed Group.

\section{CONCLUSIONS}

HDR endovaginal brachytherapy, with or without External Beam Radiation Therapy provided excellent Overall Survival (OS)/ Progression Free Survival (PFS). The toxicity profile was found to be very minimal.

\section{KEY WORDS}

Endometrial Cancer, HDR Vaginal Brachytherapy, Adenocarcinoma. External-Beam Radiotherapy (EBRT)

HOW TO CITE THIS ARTICLE: Saravanan S, Vasanthamani P. Outcome analysis of early endometrial cancers managed by adjuvant vault brachytherapy-a single institutional study. J. Evolution Med. Dent. Sci. 2019;8(29):2351-2354, DOI: $10.14260 /$ jemds/2019/515

\section{BACKGROUND}

Uterine cancer is one of the most common cancers among gynaecological cancers after cancer cervix.[1] Novel treatment in diagnosis of endometrial cancer in earlier stages have led to improved Overall Survival (OS) and Progression Free Survival (PFS).[2] Surgery in the form of a Total Abdominal Hysterectomy (TAH) and Bilateral Salpingo-Oophorectomy (BSO) with or without lymph node dissection, is the standard of care in early endometrial cancers. ${ }^{[3,4]}$ Adjuvant ExternalBeam Radiotherapy (EBRT) plays a vital role in the management of low-risk endometrial cancer with better local control rates stage IA-IB, grade [G] 1-2, FIGO 1988).[5]

'Financial or Other Competing Interest': None.

Submission 16-05-2019, Peer Review 02-07-2019,

Acceptance 09-07-2019, Published 22-07-2019.

Corresponding Author:

P. Vasanthamani,

Dean Quarters,

Kilpak, Chennai,

Tamilnadu,

India.

E-mail: drsaravanancancer@gmail.com

DOI: $10.14260 /$ jemds $/ 2019 / 515$

More so the vaginal brachytherapy (VBT) alone can be used as adjuvant treatment in intermediate-risk disease (Stage IB G1-2 disease, stage IA G3 disease and stage IC G1-2 disease), with successful results.[6,7,8,9] Addition of chemotherapy to adjuvant RT minimises the rate of distant metastasis.[10] Because of excellent local control VBT is often used. Hence we compare and evaluate overall survival (Primary endpoint ) and local control and toxicity (Secondary endpoint) in a group of patients with post-operative endometrial cancer along with adjuvant high-dose-rate (HDR) endovaginal brachytherapy (BT) or Monotherapy with endovaginal Brachytherapy (BT) (Treated Group) Vs in a group of patients managed with primary surgery alone (Observed group).

\section{METHODS}

\section{Characteristics of Patients}

This prospective analytical study included 306 patients who presented to Govt. Royapettah Hospital with pathologically documented invasive endometrial cancer in the first stage and those treated with or without brachytherapy were subjected to a controlled study in 2012-2018 after Institutional Ethical Committee Clearance. All patients underwent total abdominal hysterectomy with bilateral salpingo-oophorectomy. The patients were split into two groups as shown in Figure 1. 
1. Treated Group: who received adjuvant brachytherapy with or without External beam Radiation Therapy (EBRT)

2. Observed Group: who used only observation after surgery.

The two groups were divided into patients with low and medium risk. There were 103 low-risk and 133 medium-risk patients in the Treated Group and 32 low-risk patients and 38 medium risk patients in the Observed Group (Table 1). Patients from both groups underwent hysterectomy with or without node dissection. Patients in the treated group were irradiated by HDR (High Dose Rate) brachytherapy using Ir192 source with or without EBRT. Inclusion criteria: Patients who underwent the TAH with FIGO stage I intermediate- and high-risk endometrial carcinoma, consisting of stage Ia (grade II, grade III), stage Ib (grade I, grade II) and stage I b (grade III), respectively and KPS $\geq 70$ were included.

\section{Vaginal Brachytherapy}

The patients with endometrial cancer were presented to us either after completion of EBRT or without EBRT. The Single Vaginal applicator, a CT-compatible one was used in this study. Then cylinders of optimal diameter (Either 1.5, 2, 2.5 and 3 $\mathrm{cm})$ were inserted into the vagina based on the size of the vaginal cavity and assembly was locked. CT Simulation (Somatom AS 20 open model of Siemens CT Simulator) was done immediately followed by planning in Oncentra Treatment Planning System. (Veenendaal, the Netherlands, the Elekta). The patient underwent CT simulation for the remaining fractions and the Vaginal Brachytherapy was then delivered using Micro Selectron high-dose-rate Brachytherapy (HDR -V3 Model of Elekta) unit. In arm I, VBT was delivered after a period of 7-10 days after completion of 45 to $50 \mathrm{~Gy}$., of EBRT. The applications were done through a delivery of $6 \mathrm{~Gy}$ per fraction with a gap of 7 days in two applications. In arm II where vault brachytherapy used as monotherapy through three weekly fractions of VBT was delivered with a dose of 6 to 7 Gy per fraction.

\section{Follow Up}

Post brachytherapy patients were followed up once in every three months during the first two years and then every six months and every year MRI scan was done. The patients were then assessed for the 3-, and 5 years Overall survival (OS), Disease free survival (DFS), and LRFS (PFS) rate analysis. The second outcome was the assessment of late toxicity. Treatment failures were classified as local recurrence, loco-regional recurrence or distant metastases.

\section{Statistical Analysis}

The follow up data were analysed by mean, median, and range. All analyses were performed using the Statistical Package for Social Science (SPSS) version 22 software and Statistical survival analysis was performed with the Kaplan-Meier method and for DFS and LRFS the log-rank test was used. A statistical value of $p>0.05$ is considered more significant.
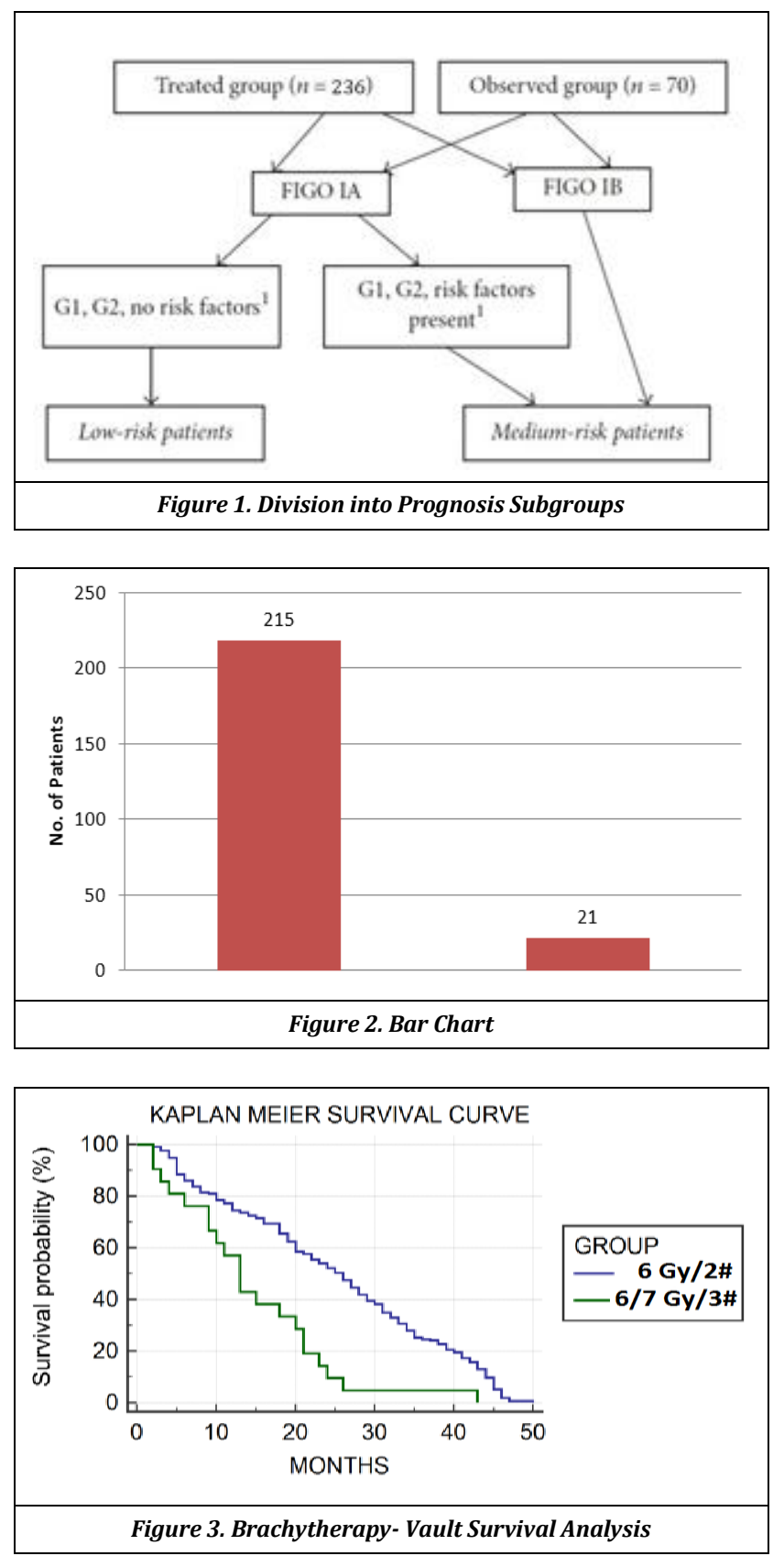

\begin{tabular}{|c|c|c|c|c|c|}
\hline Factor & Mean & SE & $\begin{array}{c}\text { 95\% CI for the } \\
\text { Mean }\end{array}$ & Median & $\begin{array}{c}\text { 95\% CI for the } \\
\text { Median }\end{array}$ \\
\hline A & 24.935 & 0.941 & 23.090 to 26.780 & 26.000 & 22.000 to 28.000 \\
\hline B & 14.571 & 2.161 & 10.336 to 18.807 & 13.000 & 9.000 to 20.000 \\
\hline Overall & 24.013 & 0.898 & 22.252 to 25.774 & 24.000 & 20.000 to 26.000 \\
\hline \multicolumn{5}{|c|}{ Mean and Median survival } \\
\hline
\end{tabular}

\begin{tabular}{|c|c|}
\hline Chi-squared & $\mathbf{1 7 . 0 7 1 1}$ \\
\hline DF & 1 \\
\hline Significance & $\mathrm{p}<0.0001$ \\
\hline \multicolumn{2}{|c|}{ Comparison of Survival Curves } \\
\hline
\end{tabular}

\begin{tabular}{|c|c|c|}
\hline Factor & A & B \\
\hline A & - & 4.2145 \\
B & 0.2373 & 2.1300 to 8.3388 \\
\hline \multicolumn{2}{|c|}{ Hazard Ratio with 95\% Confidence Interval } \\
\hline
\end{tabular}

\begin{tabular}{|c|c|c|}
\hline Demographical or Clinical & Treated Group & $\begin{array}{c}\text { Observed } \\
\text { Group }\end{array}$ \\
\hline Risk factor & $\mathrm{n}=236$ & $\mathrm{n}=70$ \\
\hline Age of patients: median (range) & $65(47-90)$ years & $66(46-90)$ years \\
\hline FIGO: & & \\
\hline IA & $192(81.3 \%)$ & $59(84 \%)$ \\
\hline IB & $44(18.6 \%)$ & $11(15.7 \%)$ \\
\hline
\end{tabular}




\begin{tabular}{|c|c|c|}
\hline Number of risk factors & & \\
\hline 0 & $103(43.6 \%)$ & $35(50 \%)$ \\
\hline 1 & $98(41.5 \%)$ & $29(41.4 \%)$ \\
\hline 2 & $35(14.8 \%)$ & $6(8.6 \%)$ \\
\hline Type of risk factors & & \\
\hline LUSI & $33(13.9 \%)$ & $2(2.9 \%)$ \\
\hline Size of tumor > 2 cm & $36(15.2 \%)$ & $2(2.9 \%)$ \\
\hline Histopathologic type II & $24(10.1 \%)$ & $13(18.5 \%)$ \\
\hline Histology & & \\
\hline Adenocarcinoma & $154(65 \%)$ & $35(50 \%)$ \\
\hline Adenocarcinoma with squamous metaplasia & $27(1.1 \%)$ & $9(12.8 \%)$ \\
\hline Clear cell 1 & $16(0.6 \%)$ & $6(8.6 \%)$ \\
\hline MMMT 1 & $17(0.72 \%)$ & $11(15.7 \%)$ \\
\hline ESS 1 & $22(0.93 \%)$ & $9(12.8 \%)$ \\
\hline Age > 70 & $137(58 \%)$ & $23(32.8 \%)$ \\
\hline Table 1. Base Line Characteristics of Patients \\
\hline \multicolumn{2}{|l}{} \\
\hline \multicolumn{2}{|c|}{} \\
\hline
\end{tabular}

\begin{tabular}{|c|c|c|c|}
\hline & 3 OS & 5 OS & p \\
\hline \multicolumn{4}{|c|}{ Group } \\
\hline Treated & $96 \%$ & $93 \%$ & \\
\hline Observed & $90 \%$ & $92.12 \%$ & \\
\hline \multicolumn{4}{|c|}{ Risk } \\
\hline Low (treated) & $100 \%$ & $100 \%$ & $\mathrm{p}=0.018$ \\
\hline Medium (treated) & $94 \%$ & $87 \%$ & \\
\hline Low (observed) & $94 \%$ & $95 \%$ & \\
\hline Medium (observed) & $92 \%$ & $82 \%$ & $\mathrm{P}>0.05$ \\
\hline \multicolumn{4}{|c|}{ Table 2a.3 OS \& 5 OS, in Treated and Observed Groups } \\
\hline
\end{tabular}

\begin{tabular}{|c|c|c|c|}
\hline & 3 -DFS & 5-DFS & \\
\hline Group & & & \\
\hline Treated & $96 \%$, & $95 \%$, & \\
\hline Observed & $91 \%$, & $92 \%$ & \\
\hline Risk & & & \\
\hline Low (treated) & $100 \%$ & $97.5 \%$ & \\
\hline Medium (treated) & $93 \%$ & $93 \%$ & \\
\hline Low (observed) & $95 \%$ & $96 \%$ & \\
\hline Medium (observed) & $93 \%$ & $93 \%$ & \\
\hline Table 2b, 3 -DFS \& 5 DFS in Treated and Observed Groups \\
\hline
\end{tabular}

\begin{tabular}{|c|c|c|c|}
\hline & 3 - LRFS & 5 - LRFS & \\
\hline Group & & & \\
\hline Treated & $97,77 \%$, & $97,77 \%$, & $\mathrm{NS}$ \\
\hline Observed & $97,14 \%$, & $95,57 \%$ & \\
\hline Risk & & & \\
\hline Low (treated) & $100 \%$ & $100 \%$ & \\
\hline Medium (treated) & $96 \%$ & $96 \%$ & \\
\hline Low (observed) & $93.75 \%$ & $93.75 \%$ & $\mathrm{NS}$ \\
\hline Medium (observed) & $100 \%$ & $97,06 \%$, & \\
\hline OS: overall survival, DFS: disease-free survival, LRFS: local relapse-free survival. \\
\hline Table 2c. 3LRFS and 5 LRFS in Treated and Observed Groups \\
\hline
\end{tabular}

\begin{tabular}{|c|c|c|c|c|c|c|c|c|}
\hline & $\begin{array}{c}\text { Treated } \\
\text { Group }\end{array}$ & $\mathbf{p}$ & $\begin{array}{c}\text { 5-OS } \\
\text { Observed } \\
\text { Group }\end{array}$ & $\mathbf{p}$ & $\begin{array}{c}\text { Treated } \\
\text { Group }\end{array}$ & $\mathbf{p}$ & $\begin{array}{c}\text { Observed } \\
\text { Group }\end{array}$ & $\mathbf{p}$ \\
\hline FIGO IA & $84,76 \%$, & $\mathrm{NS}$ & $96,11 \%$, & $\mathrm{NS}$ & $91,35 \%$ & 0.047 & $91,91 \%$ & $\mathrm{NS}$ \\
\hline FIGO IB & $82,41 \%$ & & $90,10 \%$ & & $81,71 \%$ & & $100 \%$ & \\
\hline No risk factors & $93,60 \%$ & & $100 \%$ & & $100 \%$ & & $100 \%$, & \\
\hline & & 0.15 & & $\mathrm{NS}$ & & $\mathrm{NS}$ & & $\mathrm{NS}$ \\
\hline 1 risk factor & $72,61 \%$ & & $88,14 \%$ & & $91,72 \%$ & & $88,14 \%$ & \\
\hline 2 risk factors & $56,66 \%$ & & $94 \%$ & & $90,93 \%$ & & $92,23 \%$ & \\
\hline Age $>70$ & $88,83 \%$, & & $95,29 \%$, & & $92,27 \% \mathrm{NS}$ & & $90,28 \%$ & \\
\hline Age $<70$ & $67,81 \%$ & 0.011 & $94,44 \%$ & $\mathrm{NS}$ & $85,87 \%$ & NS p $=0.1$ & $91,21 \%$ & NS \\
\hline \multicolumn{7}{|c|}{ Table 3. 5-OS and 5-DFS in Patients with Risk Factors } \\
\hline
\end{tabular}

\section{RESULTS}

\section{Survival (OS and DFS)}

In this study Patients were followed for an average of 50 months after treatment (Over 5 years). Number of patients who were disease free in the treated group were 216 (91\%) and in observed group were 54 (85\%).Number of patients with vault recurrence only $12(0.5 \%)$ in the Treated Group and $2(2.9 \%)$ in Observed Group. Patients with pelvic nodes recurrence only $10(0.4 \%)$ in Treated Group and $2(2.9 \%)$ in Observed Group. Patients with distant metastases only 12 (0.5 $\%)$ in the Treated group and 2 (2.9\%) in Observed Group. The median follow-up time in both groups was 58.88 months.

\section{Toxicity}

Acute toxicity was found in less than $20.6 \%$ in the Vault Brachy monotherapy group which received 21 Gy in $3 \#$ one week apart. Majority of those toxicities occurred in the Treated Group were of G1 - G2 in nature and self-limiting. Proctitis occurred in 15 patients $(6,3 \%)$ as G2 late morbidity. G1-G2 late vaginal toxicity was observed in 66 patients in the treated group (27.7\%). G3 - G4 late vaginal toxicity was seen in 5 patients $(2 \%$.).(6)

In Table-2 we could see, a statistically significant difference in overall survival between the low- and the medium-risk patients ( $p=0.018)$. The median time elapsed to recur was 43.37 months (range 0-90 months). Number of patients with local recurrence only $12(0.5 \%)$ Treated Group and 2 (2.9\%) in Observed Group. Number of patients with pelvic nodes recurrence only $10(0.4 \%)$ in Treated Group and $2(2.9 \%)$ in Observed Group. Number of patients with distant metastases only $12(0.5 \%)$ in the Treated Group and $2(2.9 \%)$ in Observed Group. Distant metastases were significantly found in lung and Abdominal lymph nodes. In Both Groups, majority of local recurrences were found in medium-risk patients.

\section{DISCUSSION}

The rate of recurrence was not statistically significant concluded by Sorbe et al(9) in his study of comparison between observation alone and endovaginal brachytherapy. He found in this subset of patients after the use of adjuvant brachytherapy the local recurrence is very minimal and the rate of OS and PFS was high. The older age favours deterioration of overall survival by $12 \%$.[11,12] The prognosis was found to be affected by various risk factors such as site of tumour, tumour size $>2 \mathrm{~cm}$, Bokhman tumour type II, or high grade. ${ }^{[13]}$ Brachytherapy is the highest form of conformal dose delivery with very minimal dose delivery to the critical organs(14) Sorbe et al.[9] in 2005 reported in his study the overall locoregional recurrence rate was $1.4 \%$ and the rate of local recurrence $0.7 \%$. Alektiar et al.[15] analysed 382 patients with early endometrial cancer managed with TAH followed by HDR vaginal BT through a dose of 21 Gy in 3 \# one week apart. The local control rate for 5 years was $95 \%$ and higher late toxicities were seen in 3 cases ( $0.8 \%$ ). A study by MacLeod et al.(16) revealed Five-year DFS and five-year OS were $100 \%$ and $88 \%$ for stage IA (FIGO 1988), $98 \%$ and $94 \%$ for stage IB, 100 $\%$ and $86 \%$ for stage IC, and $92 \%$ and $92 \%$ for stage IIA, respectively and the toxicity profile was very low similar to our study.

The limitation of our study was an unequal distribution of few risk factors in Treated and Observed groups and the patients were randomised based on the type of risk factors like LVSI, tumour size, the grade and histopathologic type.

\section{CONCLUSIONS}

The pelvic local control rate was found to be excellent with very low toxicity profile and was statistically significant with three- and five-year progression free survival in patients with endometrial cancers who were treated by adjuvant brachytherapy $(\mathrm{p}<0.0001)$. Based on this study, patients from low- and intermediate-risk groups of endometrial cancers should be observed after surgical treatment. The usage of adjuvant management in the form of brachytherapy should be emphasized in the low-risk group (FIGO IA, G1, 2, and no risk 
factors) as it minimises the incidence of vaginal relapses and improves OS and PFS.

\section{REFERENCES}

[1] GLOBOCAN: Cancer Incidence and Mortality Worldwide. Lyon: IARC. http://globocan.iarc.fr 2012.

[2] Sabater S, Andres I, Lopez-Honrubia V, et al. Vaginal cuffbrachy therapy in endometrial cancer - a technically easy treatment? Cancer Management and Research 2017;9:351-62.

[3] Mariani A, Dowdy SC, Cliby WA, et al. Prospective assessment of lymphatic dissemination in endometrial cancer: a paradigm shift in surgical staging. Gynecologic Oncology 2008;109(1):11-8.

[4] Keys HM, Roberts JA, Brunetto VL, et al. A phase III trial of surgery with or without adjunctive external pelvic radiation therapy in intermediate risk endometrial adenocarcinoma: a Gynecologic Oncology Group study. Gynecologic Oncology 2004;92(3):744-51.

[5] Creutzberg CL, van Putten WL, Koper PC, et al. Surgery and postoperative radiotherapy versus surgery alone for patients with stage- 1 endometrial carcinoma: a multicentre randomized trial. PORTEC Study Group. Post-Operative Radiation Therapy in Endometrial Carcinoma. The Lancet 2000;355(9213):1404-11.

[6] Blake P, Swart AM, Orton J, et al. Adjuvant external beam radiotherapy in the treatment of endometrial cancer (MRCASTEC and NCIC CTG EN.5 randomized trials): pooled trial results, systematic review and meta-analysis. The Lancet 2009;373(9658):137-46.

[7] Johnson N, Cornes P. Survival and recurrent disease after postoperative radiotherapy for early endometrial cancer: systematic review and meta-analysis. BJOG: An International Journal of Obstetrics \& Gynaecology 2007;114(11):1313-20.

[8] Kong A, Simera I, Collingwood M, et al. Adjuvant radiotherapy for stage I endometrial cancer: systematic review and meta-analysis. Annals of Oncology 2007;18(10):1595-604.
[9] Sorbe B, Nordstrom B, Maenpaa J, et al. Intravaginal brachytherapy in FIGO stage I low-risk endometrial cancer: a controlled randomized study. International Journal of Gynecol Cancer 2009;19(5):873-8.

[10] Weiss E, Hirnle P, Arnold-Bofinger H, et al. Adjuvant vaginal high-dose-rate after loading alone in endometrial carcinoma: Patterns of relapse and side effects following low-dose therapy. Gynecologic Oncology 1998;71(1):72-6

[11] Alhilli MM, Bakkum-Gamez JN, Mariani A, et al. Riskadjusted outcomes in elderly endometrial cancer patients: Implications of the contrasting impact of age on progression-free and cause-specific survival. Gynecologic Oncology 2015;138(1):133-40.

[12] Dunn EF, Geye H, Platta CS, et al. Predictive factors of recurrence following adjuvant vaginal cuff brachytherapy alone for stage I endometrial cancer. Gynecologic Oncology 2014;133(3):494-8.

[13] Aristizabal P, Graesslin O, Barranger E, et al. A suggested modification to FIGO stage I endometrial cancer. Gynecologic Oncology 2014;133(2):192-6.

[14] Arenas M, Gascon M, Rovirosa A, et al. The effect of lymphadenectomy and radiotherapy on recurrence and survival in endometrial carcinoma. Experience in a population reference centre. Reports of Practical Oncology and Radiotherapy 2014;20(1):50-6.

[15] Alektiar KM, Venkatraman E, Chi DS, et al. Intravaginal brachytherapy alone for intermediate-risk endometrial cancer. International Journal of Radiation Oncology Biology Physics 2005;62(1):111-7.

[16] McCloskey SA, Tchabo NE, Malhotra HK, et al. Adjuvant vaginal brachytherapy alone for high risk localized endometrial cancer as defined by the three major randomized trials of adjuvant pelvic radiation. Gynecologic Oncology 2010;116(3):404-7. 